\title{
Desarrollo de la colaboración en revistas de psiquiatría y salud mental en el mundo.
}

\author{
Collaboration development in journals of psychiatry and mental health in the world.
}

\section{Estimados Editores:}

En el editorial del número 3 del volumen 82 de la Revista de Neuro-Psiquiatría, Vega-Dienstmaier refiere de manera muy clara que las publicaciones científicas sobre salud mental están aumentando, siendo los siguientes países los mayor producción: a nivel mundial, Estados Unidos (artículos totales), Australia (artículos por habitante) y Países Bajos (número artículos por PBI); y en Latinoamérica, Brasil (artículos totales) y Chile (artículos per cápita) (1).

Los trastornos mentales son parte importante del concepto de Salud Mental, refiriéndose que estos problemas aumentarán en Latinoamérica dentro del plazo de 10 años; uno de ellos será la demencia, donde habrá un aumento del $47 \%$ en la prevalencia de discapacidad para dichos pacientes; además, la depresión es responsable del $8 \%$ de años vividos de manera discapacitante considerando todas las enfermedades de la región. Finalmente, en el presupuesto que se tiene para salud mental se calcula que en Centroamérica y el Caribe Latino, el $12 \%$ va dirigido a establecimientos de salud mental y el $88 \%$ a hospitales psiquiátricos, mientras en América del Sur es 35\% y $65 \%$, respectivamente (2). La crisis económica que sufrió España en el año 2008 se relacionó con un aumento de los trastornos del estado de ánimo aproximadamente en 19\%, de los trastornos de ansiedad en $8 \%$ y de los trastornos por abuso de alcohol en 5\%; paralelamente hubo un masivo consumo de fármacos antidepresivos pero no una variación en la tasa de suicidios (3).

En el Perú, gracias a parámetros bibliométricos de la base de datos SCOPUS entre los años 19962018 se calcula que se publicó unos 260 artículos con un índice $\mathrm{H}=29$ en la subcategoría "Psychiatry and Mental Health" ocupando el Perú el octavo lugar (4). Con objetivo de este artículo se extrajo del portal SCImago Journal and Country Rank (SJR), información bibliométrica de las revistas de todo el mundo y luego de Latinoamérica en la categoría "Medicine" y en la subcategoría: "Psychiatry and Mental Health", considerando la participación o colaboración internacional que tienen estas revistas, siendo ésta una medida de impacto sobre cómo se está desarrollando la equidad en investigación en países con menos recursos (5).

En los gráficos 1 y 2 se puede apreciar que la revista con mayor colaboración internacional a nivel Latinoamericano en Psiquiatría y Salud Mental en el año 2018 fue la "Revista Brasileira de Psiquiatría" con un $44,3 \%$, y a nivel mundial fue la revista de "Alzheimer's and Dementia" de Países Bajos que obtuvo un $55,8 \%$. La colaboración internacional es muy importante ya que permite la sistematización y participación en el conocimiento por parte de países que no cuentan con un sistema de salud capaz de generar investigación; además, en otras especialidades de la medicina está cobrando importancia como en el caso de Salud Pública $(6,7)$.

En el Perú las enfermedades mentales y los trastornos mentales más comunes son la ansiedad y depresión, siendo esta última prevalente en Lima Metropolitana (18,2\%), la Sierra Peruana $(16,2 \%)$ y en la Selva $(21,4 \%)$; otro acápite que no debe pasar desapercibido es la violencia contra las mujeres y específicamente contra las niñas. En un estudio realizado por la OMS las cuzqueñas eran las segundas más violentadas en todo el mundo solo después de las de Etiopia (8). En la investigación médica en general la participación de nuestro país es baja, y peor en el campo de la salud mental, aunque la Revista de Neuro-Psiquiatría desarrolla un rol muy importante para que se difunda la investigación realizada en nuestro medio. Sin embargo, entre los años 2011-2013 de 43135 artículos, el Perú solo representó el 0,06\% de la producción mundial (9). Es así que durante estos últimos años la producción científica en salud 
Desarrollo de la colaboración en revistas de psiquiatría y salud mental en el mundo.

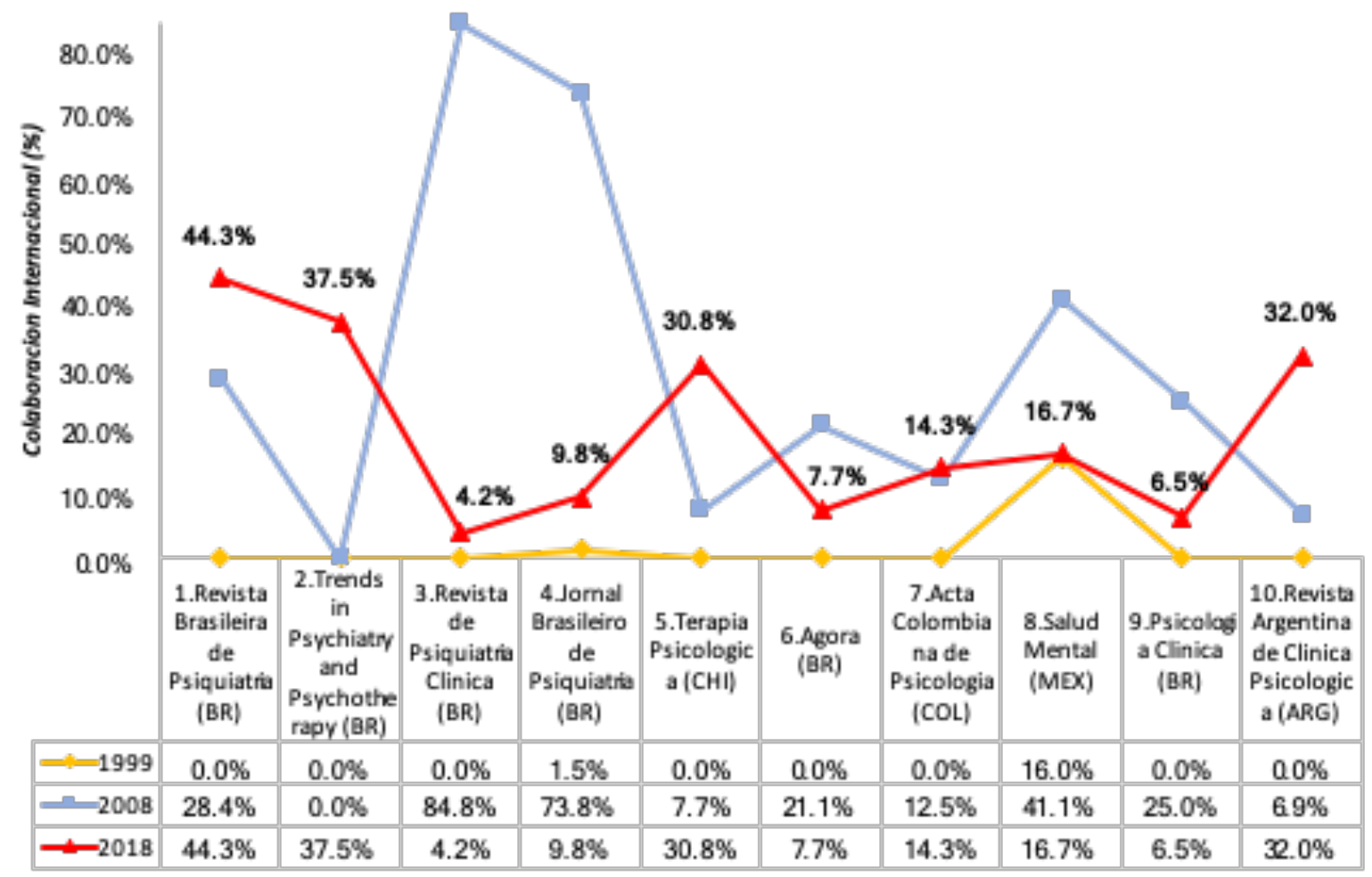

Figura 1. Colaboración internacional de las 10 mejores revistas en Latinoamérica en la subcategoría "Psychiatry and Mental Health" periodo 1999-2018. Fuente: Scimago Journal and Country Rank.

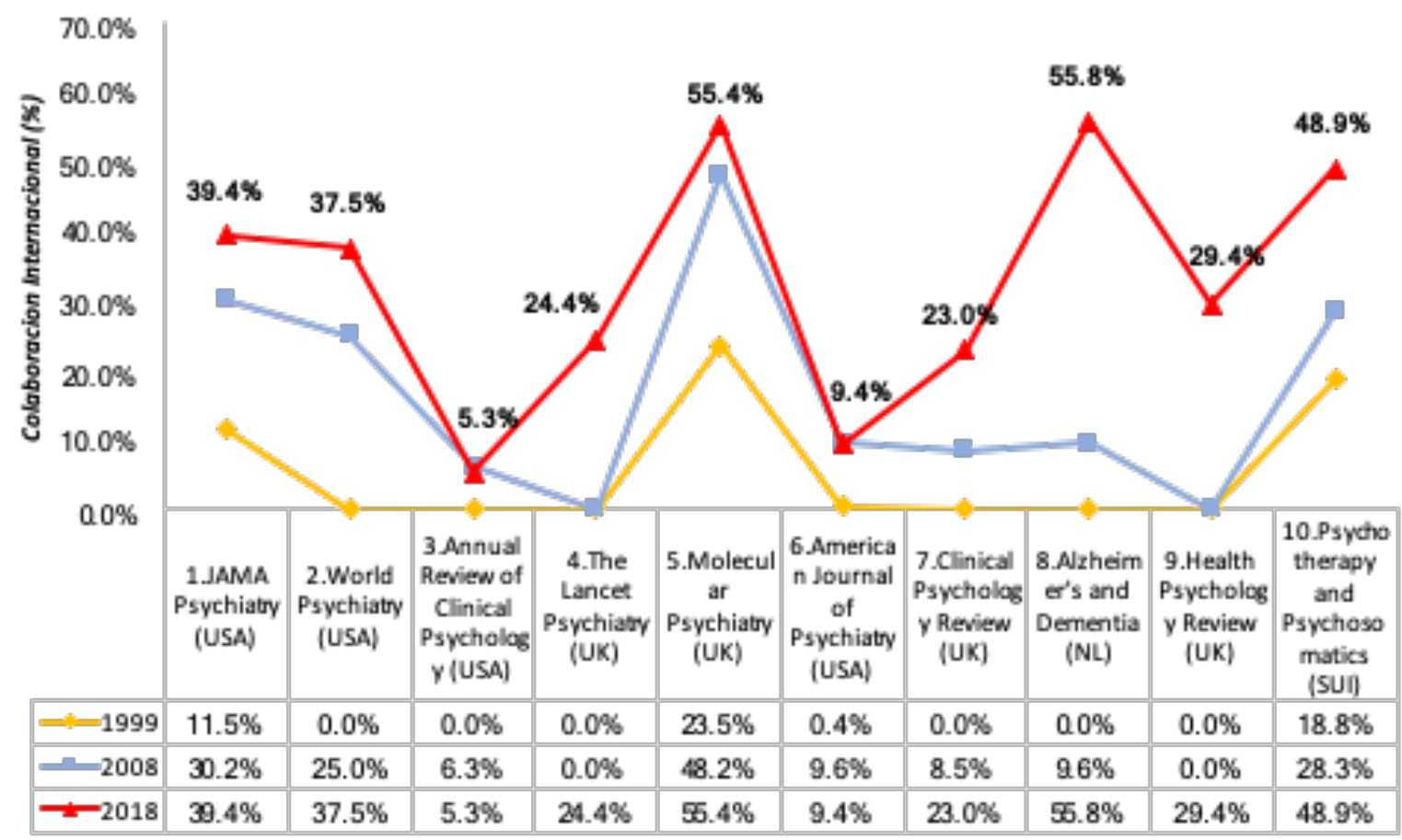

Figura 2. Colaboración internacional de las 10 mejores revistas del Mundo en la subcategoría "Psychiatry and Mental Health" periodo 1999-2018. Fuente: Scimago Journal and Country Rank. 
mental está aumentando pero requiere el apoyo de las instituciones públicas y/o privadas del país para que sea de calidad y haya regularidad en la publicación.

\section{Joel I. Atencio-Paulino ${ }^{1, b}$, Jhonatan Alcala ${ }^{1, b}$, Elmer Castañeda ${ }^{1,2, a}$, Miguel Gala ${ }^{1, a}$}

\section{Correspondencia:}

Joel Atencio.

Jr. Zorritos 1399, Block 42. Dept. 403, Cercado de Lima, Perú

Correo electrónico: yoel_005@yahoo.com

\section{REFERENCIAS BIBLIOGRAFICAS}

1. Vega-Dienstmaier JM. Publicaciones científicas peruanas sobre salud mental. Rev Neuropsiquiatr. 2019; 82(3):163-5.

2. Pan American Health Organization. Mental health in the Americas. Washington DC: Pan American Health Organization; 2017. (Citado 26 de octubre de 2019) Disponible en: https://www.paho.org/saluden-las-americas-2017/?post_t_es=la-salud-mentalen-la-region-de-las-americas
3. Gili M, García J, Roca M. Crisis económica y salud mental. Informe SESPAS 2014. Gac Sanit. 2014;28:104-8.

4. Atencio-Paulino J, Paucar-Huaman W, CondorElizarbe I. Publicación científica en especialidades de Neurología y Psiquiatría en el Perú a través del SCImago Journal and Country Rank. Rev Neuropsiquiatr. 2019;82(3):227-9.

5. Scimago. Scimago Journal \& Country Rank. Madrid: Scimago; 2019. (Fecha de acceso setiembre del 2019) Disponible en: https://www.scimagojr.com/ journalrank. php

6. Gabriel L, Bermúdez-Tamayo C. Desarrollo de la investigación para la salud en Latinoamérica y el Caribe. Colaboración, publicación y aplicación del conocimiento. Gac Sanit. 2018; 32(3):206-8.

7. Atencio-Paulino J, Condor-Elizarbe I, Paucar-Huaman W. Desarrollo de la colaboración en publicaciones sobre salud pública en Latinoamérica y el Caribe. Gac Sanit. 2019. DOI: 10.1016/j.gaceta.2019.07.004

8. Rondón M. Salud mental: un problema de salud pública en el Perú. Rev Peru Med Exp Salud Publica. 2006;23(4):237-8.

9. Luna-Solís Y. Producción científica en salud mental en Perú: Reto en tiempos de reforma de salud. Acta Méd Peru. 2015;32(1):36-40.

Facultad de Medicina Humana, Universidad Nacional del Centro del Perú. Huancayo, Perú.

2 Red de Salud de Satipo-Junín. Junín, Perú.

a Médico-Cirujano, ${ }^{\mathrm{b}}$ Estudiante de Medicina. 